environments reported poor experiences of social interaction when in hospital.

Conclusions Poor experiences of AYA receiving care in adult care settings has been identified in previous research. The findings in this present study have compared these experiences against care received in bespoke AYA care settings: advocating for the provision of dedicated spaces which bring young people together. Services must be skilfully developed to create optimal spaces to deliver holistic, age-appropriate care. This will assist the structure, design and commissioning of AYA health services that are fit for purpose.

\section{P80 CHARACTERISATION OF POPULATION, REVIEW OF SERVICE PROVISION, AND OUTCOMES FOR YOUNG PEOPLE WITH CHRONIC FATIGUE SYNDROME IN A TERTIARY CARE INPATIENT SETTING}

M Doukrou*, S Hiremath, J Begent, TY Segal. Paediatric and Adolescent, University College Hospital NHS FT, London, UK

\subsection{6/bmjpo-2019-RCPCH-SAHM.81}

Background The management of Chronic Fatigue Syndrome/ Encephalomyelitis (CFS/ME) in adolescents is challenging, especially in severe cases. There is little published evidence on best treatment, nor any agreed standard of inpatient care. Our tertiary service in a teaching hospital offers a variety of tailored options for treatment.

Aims To characterise our population of moderate to severely affected young people with CFS who were admitted to our tertiary adolescent rehabilitation service. We will describe the medical and therapeutic interventions and their impact on outcomes at discharge.

Methods A retrospective review of the medical records of 27 patients that were admitted (ranging from a day to week long stays) for rehabilitation during 2015. Data collected included patients demographics and specific treatment. Outcomes were measured in mobility, sleep and education.

Results Of the 27 study participants, 67\% were females, 29\% males and one transgender. The mean age was $15 \mathrm{y} 2 \mathrm{~m}$ (range $9 \mathrm{y} 2 \mathrm{~m}$ to $17 \mathrm{y} 7 \mathrm{~m}$ ). The mean age at discharge was $18 \mathrm{y}$ (range $15 \mathrm{y} 11 \mathrm{~m}$ to $20 \mathrm{y} 3 \mathrm{~m})$. All patients had multiple diagnoses which included CFS (85\%), chronic pain (66\%), functional gastrointestinal disorders (40\%) and mental health disorders (55\%). A flexible combination of treatment modalities (Occupational Therapy, Physiotherapy, Psychology, Child Psychiatry and Paediatrics) were offered via a series of day care (38\%), week long admissions $(31 \%)$ or both $(31 \%)$ for rehabilitation. Mobility was improved in $81 \%$ of cases, sleep in $37 \%$ and educational outcomes 74\%. 78\% showed improvement in more than one area.

Conclusions Our tertiary multidisciplinary service treats young people with chronic fatigue syndrome and other physical and psychological comorbidities. Following regular day care/inpatient admissions, we were able to demonstrate improvement in mobility and or education in $>74 \%$ of our patients. Further work is needed to define quantitative markers of positive outcomes, in order to assess the relative efficacy of different treatment options. 